\title{
A Weighted Duality based Formulation of MIMO Systems
}

\author{
Ahmad Kamal Hassan*, Muhammad Moinuddin ${ }^{* *}$, and Ubaid M. Al-Saggaf **** \\ Centre of Excellence in Intelligent Engineering Systems (CEIES), King Abdulaziz University, P.O. Box 80200, \\ Jeddah 21589, KSA \\ Department of Electrical and Computer Engineering, King Abdulaziz University,P.O. Box 80200, Jeddah \\ 21589, KSA
}

\begin{abstract}
This work is based on the modeling and analysis of multiple-input multiple-output (MIMO) system in downlink communication system. We take into account a recent work on the ratio of quadratic forms to formulate the weight matrices of quadratic norm in a duality structure. This enables us to achieve exact solutions for MIMO system operating under Rayleigh fading channels. We outline couple of scenarios dependent on the structure of eigenvalues to investigate the system behavior. The results obtained are validated by means of Monte Carlo
\end{abstract} simulations.

Keywords: Duality, MIMO, Rayleigh Fading, Antenna Diversity.

\section{INTRODUCTION}

In the design of communication networks, it is highly significant to characterize the key performance indicators (KPI) such as outage probability [1] and ergodic capacity [2] in order to understand the behavior of the cellular systems [3-4]. In doing so, the optimization of the cellular network can be done and hence the capital expenditure and operational expenditure [5] can be minimized to nonnegligible extents.

A downlink communication network consists of several base station nodes which are connected wireless with the user equipment in a nondeterministic nature. The literature in the wireless communication systems points to numerous channel models [6],we however use the Rayleigh channel herein. In this work, we model the downlink end of a communication network and perform the analysis from a user perspective. Previously, the downlink setup has been investigated to great depthssuch as in the work of [7], we howeverdeal with a more specific setup in which we use the duality structure of quadratic norms to simplify the signal-tointerference-plus-noise ratio (SINR). The proposed work considers the interference from the other users which are also connected with the same base station as well as the noise which is encountered at the receiver end. The novelty of our work is basically in formulating the design/model of a MIMO system usinga duality form and hence in such a way that we can incorporate the recent resultsbased on the residue theory approach (RTA) given in [8]. Doing so also results in the exact expressions which are in in the form of eigenvalues, Heaviside function $u($.$) , and$ exponential functions. The work also briefly discusses the basic mathematical blocks which are based on the SINR metric. In results, we touch upon two different yet interesting information theoretic cases, namely, the distinct eigenvalue case and the repeated eigenvalue case in order to understand their implications on the system. Possible extension of this work can be achieved by including the system automation structure models based on the works [911] which can result in improving the significant KPIs of downlink communication system.

The formulation of this paper is such that after Introduction in Section 1, we define the downlink communication system in Section II. Section III, deals with the notion of quadratic forms and further simplifies the SINR expression. Section IV, outlines some useful metrics which can be achieved by means of the SINR expression, whereas in Section V, the analysis part of this work is considered. Results are given in Section VI which are based on two different information theoretic perspectives, this is followed by Conclusions and References.

\section{SYSTEM MODEL}

This work is based on one of a well-known system designs put forward in [12]. It uses a downlink multi-user MIMO, i.e., MU-MIMO setup in which there are several users in the vicinity of a given base station. We have considered herein a single base station scenario in which the base station has Nnumber of transmit antenna elements and it is communicating with the Knumber of users simultaneously, further each user has Mnumber of antenna elements on its structural deck.

The SINRfor such a setup has a rather simpler expression which is given by 


$$
\operatorname{SINR}_{i}=\frac{\left\|H_{i} w_{i}\right\|^{2}}{M_{i} \sigma_{i}^{2}+\sum_{k=1, k \neq i}^{K}\left\|H_{i} w_{k}\right\|^{2}}
$$

where $\sigma_{i}^{2}$ represents the noise variance for $i$ th user, $H_{i}$ is the channel at $i$ th user, and $w_{i}$ is the transmit beamvector for the $i$ th user.

The transmit beamforming vector $\left(w_{i}{ }^{\prime} s\right)$ are orthogonal; thus, satisfying the following conditions

$$
\begin{aligned}
& w_{i} * w_{i}^{\prime}=\frac{1}{k} \mathbf{I} \\
& w_{i} * w_{j \neq i}^{\prime}=0
\end{aligned}
$$

where I represents the identity matrix.

\section{SIMPLIFICATION OF SINR EXPRESSION}

In this section, the aim is to simplify the SINR expression given in (1). Fromthe numerator term in (1), we can utilize (2)-(3) to show

$$
\begin{aligned}
& H_{i} w_{i} \\
& =\left[\begin{array}{ccc}
h_{i}^{(1,1)} w_{\mathrm{i} 1}+h_{i}^{(1,2)} w_{i 2}+ & \cdots & +h_{i}^{(1, N)} w_{i 1 N} \\
\vdots & \ddots & \vdots \\
h_{i}^{(M, 1)} w_{i 1}+h_{i}^{(M, 2)} w_{i 2}+ & \cdots & +h_{i}^{(M, N)} w_{i 1 N}
\end{array}\right] \\
& =\left[\begin{array}{c}
h_{i 1} \\
\vdots \\
h_{i M}
\end{array}\right] \\
& =h_{i}
\end{aligned}
$$

where the composite channel is a complex Gaussian channel, i.e., $h_{i} \sim C N\left(0, R_{1}\right)$, whereas $R_{1}$ is the channel correlation of the desired signal part.

Similarly, the interference terms in the denominatorof (1) can be expressed ash $h_{k}^{(k, 1)} \sim C N\left(0, R_{2}\right)$, whereas $R_{2}$ is the channel correlation of the interference part.

Now, transforming both $h_{i}$ and $h_{k}$ on to a bigger vector with dimension $1 X K M$, i.e.,

$$
h=\left[\begin{array}{llll}
h_{1}^{T} h_{2}^{T} & \ldots & h_{i-1}^{T} h_{i}^{T} h_{i+1}^{T} h_{k-1}^{T} h_{k}^{T}
\end{array}\right]
$$

and by using the notion of weighted norm, we express the signal and interference parts of (1) as follows

$$
\begin{aligned}
& \left\|h_{i}\right\|^{2}=\|h\|_{A_{i}}^{2} \\
& \left\|h_{k}\right\|^{2}=\|h\|_{A_{k}}^{2}
\end{aligned}
$$

As a result, the SINR give in (1) can be formulated as follows

$$
\operatorname{SINR}_{i}=\frac{\|h\|_{A_{i}}^{2}}{M_{i} \sigma_{i}^{2}+\|h\|_{A_{k}}^{2}}
$$

where the elements of $A_{i}$ are dual with elements of $A_{k}$ such that if

$$
\left.A_{i}=\left[\begin{array}{llll}
0 & 0 & \ldots & 0
\end{array}\right] \ldots\left[\begin{array}{llll}
1 & 1 & \ldots & 1
\end{array}\right]_{1 X M}^{i t h} \ldots\left[\begin{array}{llll}
0 & 0 & \ldots & 0
\end{array}\right]\right]_{1 X K M} .
$$

then its duality is having the following form

$$
\begin{aligned}
& A_{k}= \\
& \left.\left[\begin{array}{llll}
1 & 1 & \ldots & 1
\end{array}\right] \ldots\left[\begin{array}{llll}
0 & 0 & \ldots & 0
\end{array}\right]_{1 X M}^{i^{t h}} \ldots\left[\begin{array}{llll}
1 & 1 & \ldots & 1
\end{array}\right]\right]_{1 X K M}
\end{aligned}
$$

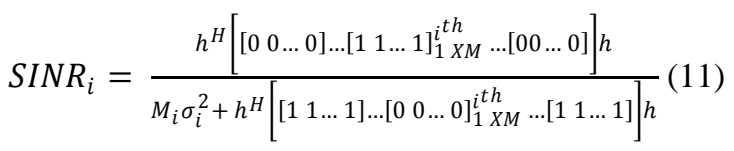

\section{KPI RELATED TO SINR}

In this section, we show the use of the formulation of SINR expression given in (11) to calculate the significant KPIs of a downlink communication system. In what follows, we list down some of the key performance indicators that can be extracted out using the aforementioned simplification.

\section{A. Outage Probability}

Outage probability is a scenario that is indicative of the loss of service at the user terminal. It is based on a defined threshold $(\xi)$, hence if the received signal is below such level, an outage occurrence incurs. Mathematically, the Outage Probability for the $i$ th user is given by

OutageProbability $_{i}=\operatorname{Pr}\left\{\operatorname{SINR}_{i}<\xi\right\} \quad$ (12)

It is noteworthy that the above form is simply the cumulative distribution function (CDF) of SINR.

\section{B. Coverage Probability}

Converge probability is related to the Outage Probability. It is defined as follows CoverageProbability $_{i}=1-\operatorname{Pr}\left\{\operatorname{SINR}_{i}<\xi\right\}(12)$ The above form is the complementary cumulative distribution function (CCDF) of SINR.

\section{Ergodic Capacity}

The ergodic capacity measured in bits/sec/Hz and it is also related with the SINR by means of the following expression.

$$
\text { ErgodicCapacity }=\boldsymbol{E}\left[\log _{2}(1+\operatorname{SINR})\right](13)
$$

\section{ANALYSIS OF SINR}

In this section, we focus on the usability of the simplified SINR form given in (8)-(11). Now by considering the Fourier representation of the Heaviside function and considering the Rayleigh fading channel, the procedure of analysis in [8] can be used to achieve the Outage Probability as

$$
\mathrm{P}\left\{\operatorname{SINR}_{i}<\xi\right\}=\int_{-\infty}^{\infty} \frac{1}{|I+\boldsymbol{A}(j w+\beta)|} \frac{e^{M_{i} \sigma_{i}^{2} \xi(j w+\beta)}}{(j w+\beta)} d w(14)
$$
where|.| represent the determinant operator, the coefficients $(j w+\beta)$ appear form the Heaviside function, and $\mathbf{A}$ is a Hermitian matrix which is defined as follows

$$
\boldsymbol{A}=(\xi+1) \boldsymbol{\Lambda}^{\frac{1}{2}} \boldsymbol{A}_{i} \Lambda^{\frac{1}{2}}-\xi \boldsymbol{\Lambda}
$$

and similarly, other parameters are defined accordingly as

Eventually, the SINR can be reformulated as 


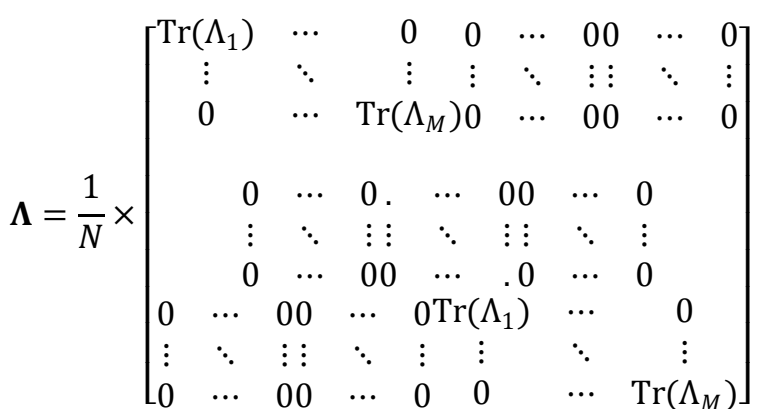
moreover, the implicit parameters in the big matrix are found as

$$
\Lambda_{j}=\boldsymbol{E}\left[h_{j}{ }^{1} \times h_{j}{ }^{1 T}\right] ; \quad \operatorname{Tr}\left(\Lambda_{j}\right)=\mathrm{N}
$$

The advantage of such formulation isthat it allows us to use the RTA methodology, the exact solution therefore can be achieved which isin a similar form as in [expression (12) - (13); [8]] however not reported herein to avoid duplication. It is noteworthy that the above formulation is for the Outage probability and therefore the Coverage probability can also be achieved by means of (12).

\section{RESULTS AND DISCUSSIONS}

The goal of this section is to understand the behavior of outage scenario as thedefined threshold

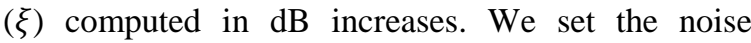
variance to $30 \mathrm{dBm}$, transmit antenna elements to 4 , and total number of users to 8 . We thereby test our formulation for 1000 channel realizations, our findings are reported in Fig. 1. In the first setup, i.e., in Fig. 1(a), a case of same eigenvalues of matrix Ain

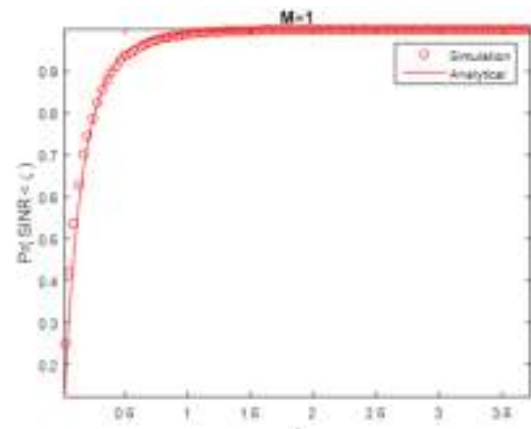

(a)

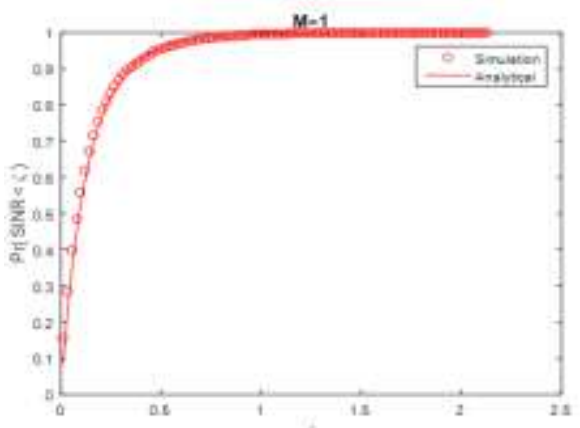

(c)
(15) is considered whereas the user whose performance is realized has a single antenna element. The outage probability increases as the bar for threshold level is increased and both the analysis results and the Monte Carlo simulation results have the CDF bounds of values between 0 and 1 . Next, in Fig. 1(b), a case of same eigenvalues of matrix $\mathbf{A}$ in (15) is considered however the number of receive elements has been increased to 2 . The performance has improved in this case as compared with the previous case. Further, in Fig. 1(c)-(d), a case of distinct eigenvalues of matrix $\mathbf{A}$ in (15) is considered for receive antenna elements set to 1 and 2 respectively. Here it can be observed that the results are comparable with our previous findings. In all cases, it is evident that the simulation results are superimposed on the analytical results hence validating our findings.

\section{CONCLUSION}

In this paper, we have outlined a system model design based on the duality structure of weighted quadratic norms which resulted in a very simple form for the SINR expression. The formulation obtained is then shown to be useful in several of the KPIs in a downlink communication system operating in a Rayleigh fading environment. We have also indicated the usability of residue theory to achieve the exact solutions. Our analysis point to the effective use of receiver diversity in a multi-user environment.

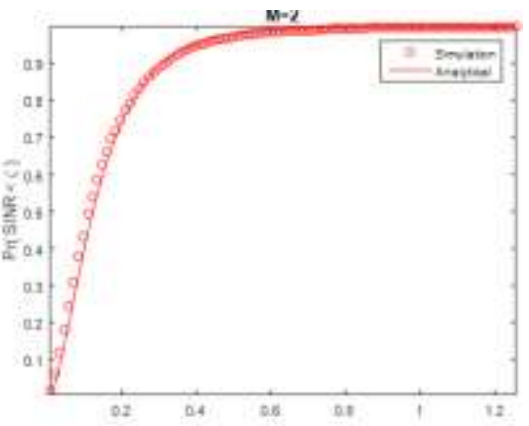

(b)

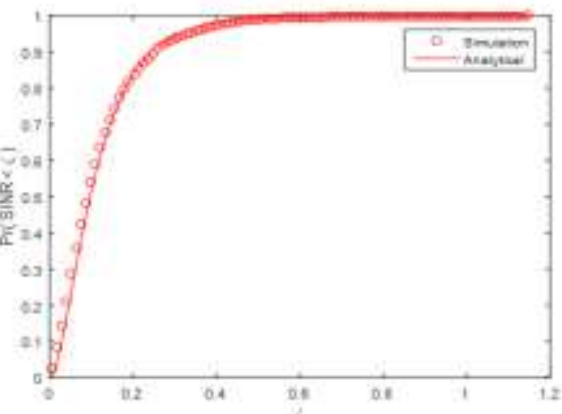

(d)

Figure 1: Analysis of Outage Probability (bold-line in 'red') and validation by means of Monte Carlo simulations(hollow circles in 'red'). 


\section{REFERENCES}

[1]. A.K. Hassan, M. Moinuddin, and U. M. AlSaggaf, 2016, August. Beamforming in Massive MU-MIMO cellular networks: A stochastic geometry approach.6th International Conference on Intelligent and Advanced Systems (ICIAS) (pp. 1-5) IEEE.

[2]. L. Li and A. J. Goldsmith, 2001. Capacity and optimal resource allocation for fading broadcast channels. I. Ergodic capacity. IEEE Transactions on Information Theory, 47(3), pp.1083-1102.

[3]. S. H. Lee, 1980. Performance Indexes of a Telecommunication network. IEEE Trans. Reliability, pp.24-26.

[4]. A. M. Rushdi, 1988. Performance indexes of a telecommunication network. IEEE transactions on reliability, 37(1), pp.57-64.

[5]. H. ElSawy, E. Hossain, andM. Haenggi, 2013. Stochastic geometry for modeling, analysis, and design of multi-tier and cognitive cellular wireless networks: A survey. IEEE Communications Surveys \& Tutorials, 15(3), pp.996-1019.

[6]. R. B. Ertel, P. Cardieri,K. W. Sowerby, T. S. Rappaport, and J. H. Reed, 1998. Overview of spatial channel models for antenna array communication systems. IEEE personal communications, 5(1), pp.10-22.

[7]. S. Hara and R. Prasad, 1997. Overview of multicarrier CDMA. IEEE communications Magazine, 35(12), pp.126-133.

[8]. T.Y. Al-Naffouri, and B. Hassibi, 2009, June. On the distribution of indefinite quadratic forms in Gaussian random variables. In Information Theory, 2009. ISIT 2009. IEEE International Symposium on (pp. 1744-1748). IEEE.

[9]. A.A. Mulla and P. N. Vasambekar, 2016. Overview on the development and applications of antenna control systems. Annual Reviews in Control, 41, pp.47-57.

[10]. H. Monga, Automated Alignment of Microwave Antenna of Base Transceiver Station by utilizing hybrid sources.

[11]. M.A. Hoque and A. K. Hassan, 2015. Modeling and performance optimization of automated antenna alignment for telecommunication transceivers. Engineering Science and Technology, an International Journal, 18(3), pp.351-360.

[12]. M. Sadek, A.Tarighat, and A. H. Sayed, 2007. Active antenna selection in multiuser MIMO communications. IEEE
Transactions on Signal Processing, 55(4), pp.1498-1510. 